ARTICLE OPEN

\title{
Entropy generation in Gaussian quantum transformations: applying the replica method to continuous-variable quantum information theory
}

\author{
Christos N Gagatsos ${ }^{1}$, Alexandros I Karanikas ${ }^{2}$, Georgios Kordas ${ }^{2}$ and Nicolas J Cerf ${ }^{1}$
}

In spite of their simple description in terms of rotations or symplectic transformations in phase space, quadratic Hamiltonians such as those modelling the most common Gaussian operations on bosonic modes remain poorly understood in terms of entropy production. For instance, determining the quantum entropy generated by a Bogoliubov transformation is notably a hard problem, with generally no known analytical solution, while it is vital to the characterisation of quantum communication via bosonic channels. Here we overcome this difficulty by adapting the replica method, a tool borrowed from statistical physics and quantum field theory. We exhibit a first application of this method to continuous-variable quantum information theory, where it enables accessing entropies in an optical parametric amplifier. As an illustration, we determine the entropy generated by amplifying a binary superposition of the vacuum and a Fock state, which yields a surprisingly simple, yet unknown analytical expression.

npj Quantum Information (2015) 2, 15008; doi:10.1038/npjqi.2015.8; published online 16 February 2016

\section{INTRODUCTION}

Gaussian transformations are ubiquitous in quantum physics, playing a major role in quantum optics, quantum field theory, solid-state physics or black-hole physics. ${ }^{1}$ In particular, the Bogoliubov transformations resulting from Hamiltonians that are quadratic (bilinear) in mode operators are among the most significant Gaussian transformations, well known to model superconductivity $^{2}$ but also describing a much wider range of physical situations, from squeezing or amplification in the context of quantum optics ${ }^{3-6}$ to Unruh radiation in an accelerating frame $e^{7-9}$ or even Hawking radiation as emitted by a black hole. $^{10-12}$ In the present article, we focus on Gaussian bosonic transformations, which are at the heart of so-called Gaussian quantum information theory. ${ }^{13}$ These transformations encompass the passive coupling between modes of the electromagnetic field as effected by a beam splitter in bulk optics or an optical coupler in fibre optics, as well as the active transformations resulting from parametric downconversion in a nonlinear optical medium, which are traditionally used as a source of quantum entanglement.

Although they are common, quantum Gaussian processes are poorly understood in terms of entropy generation. Indeed, the symplectic formalism in phase-space representation is not suited to calculate von Neumann entropies as this requires diagonalising density operators in state space. ${ }^{14}$ When amplifying an optical state using parametric downconversion, for example, the output state suffers from quantum noise, which is an increasing function of the amplification gain. ${ }^{15}$ Characterising this noise in terms of entropy is indispensable for determining the capacity of Gaussian bosonic channels. ${ }^{16-18}$ However, the output entropy is not accessible for an arbitrary input state because it is hard-usually impossible-to diagonalise the corresponding output state in infinite-dimensional Fock space. With the notable exception of
Gaussian states (e.g., the vacuum state, resulting after amplification in a thermal state of well-known entropy), very few analytical results are available as of today. ${ }^{18}$

In this article, we demonstrate that the replica method can be successfully exploited to overcome this central problem and find an exact analytical expression for the entropy generated by Gaussian processes acting on non-trivial bosonic states. The replica method is known to be an invaluable tool in statistical physics, especially with disordered systems, ${ }^{19}$ and in quantum field theory. ${ }^{20}$ Here we first apply it to continuous-variable (Gaussian) quantum information theory and show that it enables accessing the entropy generated by a quantum optical amplifier, opening a new way towards the entropic characterisation of Gaussian channels as considered in quantum communication theory. To illustrate the power of this approach in an interesting and experimentally relevant case, we investigate the output entropy when amplifying a binary superposition of the vacuum and an arbitrary Fock state, which yields a surprisingly simple analytical expression.

\section{RESULTS}

Gaussian bosonic transformations

The linear coupling between two modes of the electromagnetic field with, e.g., a beam splitter, is modelled by the (passive) quadratic Hamiltonian $H=i \hat{a} \hat{b}^{\dagger}-i \hat{a}^{\dagger} \hat{b}$, where $\hat{a}$ and $\hat{b}$ are bosonic mode operators. This operation can be shown to preserve the Gaussian character of a quantum state, or more precisely the quadratic exponential form of its characteristic function. The corresponding transformation in phase space is the rotation $\hat{a} \rightarrow \cos \theta \hat{a}+\sin \theta \hat{b}$ and $\hat{b} \rightarrow \cos \theta \hat{b}-\sin \theta \hat{a}$, where $\cos ^{2} \theta$ is the transmittance. Another generic Gaussian transformation

\footnotetext{
${ }^{1}$ Quantum Information and Communication, Ecole polytechnique de Bruxelles, Université libre de Bruxelles, Brussels, Belgium and ${ }^{2}$ Nuclear and Particle Physics Section, Physics Department, University of Athens, Athens, Greece.

Correspondence: NJ Cerf (ncerf@ulb.ac.be)

Received 17 March 2015; revised 18 June 2015; accepted 1 August 2015
} 
results from parametric downconversion in a nonlinear medium which is modelled by the (active) quadratic Hamiltonian $H=i \hat{a} \hat{b}-i \hat{a}^{\dagger} \hat{b}^{\dagger}$. It effects the Bogoliubov transformation $\hat{a} \rightarrow \cosh r \hat{a}+\sinh r \hat{b}^{\dagger}$ and $\hat{b} \rightarrow \cosh r \hat{b}+\sinh r \hat{a}^{\dagger}$, where $\cosh ^{2} r$ is the parametric amplification gain. More generally, the set of all linear canonical transformations effected by quadratic bosonic Hamiltonians, also referred to as symplectic transformations, can easily be characterised in terms of affine transformations in phase space (e.g., rotations and area-preserving squeeze mapping).

\section{Accessing the generated entropy}

Calculating the von Neumann entropy $S(\hat{\rho})=-\operatorname{tr}(\hat{\rho} \ln \hat{\rho})$ of a bosonic mode that is found in state $\hat{\rho}$ at the output of a Gaussian transformation is often an intractable task because it requires turning to state space and finding the infinite-size vector of eigenvalues of $\hat{\rho}$. The above symplectic formalism for Gaussian transformations is useless in this respect, albeit for Gaussian states. However, this problem can be circumvented by adapting the replica method, which relies on expressing $\operatorname{tr}\left(\hat{\rho}^{n}\right)$ as a function of the number of replicas $n \in \mathbb{N}^{*}$ and taking the derivative at $n=1$, thereby avoiding the need to diagonalise $\hat{\rho}$ (see Materials and Methods). As we shall show, dealing with a quadratic Hamiltonian makes the replica method a perfect tool to access von Neumann entropies because it involves Gaussian integrations, or else some tricks can be used in order to bring $\operatorname{tr}\left(\hat{\rho}^{n}\right)$ to a calculable form

First, we illustrate this principle with a generic zero-mean rotation-invariant Gaussian state, namely a thermal state $\hat{\rho}_{0}=$ $\left(1-|\tau|^{2}\right) \sum_{k=0}^{\infty}|\tau|^{2 k}|k\rangle\langle k|$ characterised by a mean photon number $N \equiv|\tau|^{2} /\left(1-|\tau|^{2}\right)$. As $\hat{\rho}_{0}$ is in a diagonal form, it is of course straightforward to calculate its entropy, giving the well-known formula $S\left(\hat{\rho}_{0}\right)=g(N) \equiv(N+1) \log (N+1)-N \log N$. However, we may also start with its non-diagonal representation in the coherent-state basis $\{|a\rangle\}$, where $a$ is a complex number, namely

$$
\hat{\rho}_{0}=\frac{1}{\pi} \frac{1-|\tau|^{2}}{|\tau|^{2}} \int \mathrm{d}^{2} a e^{-\frac{1-|\tau|^{2}}{|\tau|^{2}}|a|^{2}}|a\rangle\langle a|
$$

By making the change of variable $a \rightarrow|\tau| a$ and using $\langle a \mid \beta\rangle=e^{-\left(|\alpha|^{2}+|\beta|^{2}-2 \alpha^{*} \beta\right) / 2}$, we can write

$$
\operatorname{tr}\left(\hat{\rho}_{0}^{n}\right)=\frac{\left(1-|\tau|^{2}\right)^{n}}{\pi^{n}} \int \mathrm{d}^{2} a_{1} \ldots \int \mathrm{d}^{2} a_{n} e^{-\bar{a}^{\dagger} M \bar{a}}
$$

where $\bar{a}=\left(a_{1}, \ldots a_{n}\right)^{T}$ is a column vector and $M$ is the $n \times n$ circulant matrix

$$
M=\left(\begin{array}{ccccc}
1 & -|\tau|^{2} & 0 & \ldots & 0 \\
0 & 1 & -|\tau|^{2} & \ldots & 0 \\
\vdots & \vdots & \vdots & \ddots & \vdots \\
-|\tau|^{2} & 0 & 0 & \ldots & 1
\end{array}\right)
$$

Equation (2) is a simple Gaussian integral, which, using the determinant det $M=1-|\tau|^{2 n}$, can be expressed as

$$
\operatorname{tr}\left(\hat{\rho}_{0}^{n}\right)=\frac{\left(1-|\tau|^{2}\right)^{n}}{1-|\tau|^{2 n}}
$$

Applying the replica method, we readily find that

$$
-\left.\frac{\partial}{\partial n} \operatorname{tr}\left(\hat{\rho}_{0}^{n}\right)\right|_{n=1}=\ln \frac{1}{1-|\tau|^{2}}+\frac{|\tau|^{2}}{1-|\tau|^{2}} \ln \frac{1}{|\tau|^{2}}
$$

which coincides with the above expression $S\left(\hat{\rho}_{0}\right)=g(N)$ for the entropy of a thermal state, as expected.

\section{Amplifying a Fock state}

Next, we consider the problem of expressing the entropy $S_{m}$ generated by amplifying an arbitrary Fock state $|m\rangle$. For this, we consider a two-mode squeezer of parameter $\xi=|\xi| e^{i \phi}$, applying the unitary transformation

$$
\hat{U}=e^{-\xi \hat{a}^{\dagger} \hat{b}^{\dagger}+\xi^{*} \hat{a} \hat{b}}
$$

on the initial state $|m\rangle_{a}|0\rangle_{b}$ (subscript $a$ refers to the signal mode, while $b$ refers to the idler mode). The reduced output state $\hat{\rho}_{m}$ of the signal mode is diagonal in the Fock basis, with a vector of eigenvalues given by ${ }^{18,21}$

$$
p_{k}^{(m)}=\left(1-|\tau|^{2}\right)^{m+1}\left(\begin{array}{c}
k+m \\
k
\end{array}\right)|\tau|^{2 k}, \quad k \in \mathbb{N}
$$

where $\tau=\tanh \xi$, from which we find

$$
\operatorname{tr}\left(\hat{\rho}_{m}^{n}\right)=\left(1-|\tau|^{2}\right)^{n(m+1)} \sum_{k=0}^{\infty}\left(\begin{array}{c}
k+m \\
k
\end{array}\right)^{n}|\tau|^{2 n k} .
$$

Equation (8) can be re-expressed in a closed form as

$$
\operatorname{tr}\left(\hat{\rho}_{m}^{n}\right)=\frac{\left(1-|\tau|^{2}\right)^{n(m+1)}}{|\tau|^{2 n}} \mathrm{Li}_{-n}^{(m)}\left(|\tau|^{2 n}\right) .
$$

where

$$
\mathrm{Li}_{-n}^{(m)}(\zeta) \equiv \sum_{k=0}^{\infty}\left(\begin{array}{c}
k+m \\
k
\end{array}\right)^{n} \zeta^{k+1}
$$

and $\mathrm{Li}_{-n}^{(1)}(\zeta)$ denotes the polylogarithm of order $-n .^{22}$ Applying the replica method to Equation (9) and taking into account $\mathrm{Li}_{-1}^{(m)}(\zeta)=\zeta /(1-\zeta)^{m+1}$, we obtain the entropy

$$
\begin{aligned}
S_{m} & =-\left.\frac{\partial}{\partial n} \operatorname{tr}\left(\hat{\rho}_{m}^{n}\right)\right|_{n=1} \\
& =\ln \frac{|\tau|^{2}}{\left(1-|\tau|^{2}\right)^{m+1}}-\left.\frac{\left(1-|\tau|^{2}\right)^{m+1}}{|\tau|^{2}} \frac{\partial}{\partial n} \operatorname{Li}_{-n}^{(m)}\left(|\tau|^{2 n}\right)\right|_{n=1} .
\end{aligned}
$$

Using $\mathrm{Li}_{-n}^{(0)}(\zeta)=\zeta /(1-\zeta)$, it is easy to check that Equation (11) gives the correct value for $S_{0}$, that is, the entropy of a thermal state $\hat{\rho}_{0}$ as in Equation (5). Interestingly, as the polylogarithm $\mathrm{Li}_{-n}^{(1)}(\zeta)$ is a well-studied function, a closed expression can also be found for $S_{1}$ in terms of Eulerian numbers (Supplementary Information). For $m>1$, the function $\mathrm{Li}_{-n}^{(m)}(\zeta)$ assumes a summation form, which is convergent and differentiable with respect to $n$, yielding an analytical expression for $S_{m}$ (Supplementary Information).

\section{Amplifying a superposition of Fock states}

Now, we show that the same procedure makes it possible to express the entropy in situations where no diagonal form is available for the output state, so the replica method becomes essential. As an illustration, consider the amplification of a binary superposition of the type

$$
|\psi\rangle=\frac{|0\rangle+z|m\rangle}{\sqrt{1+z^{2}}}
$$

where we take $z \in \mathbb{R}$ without loss of generality. By using the BakerCampbell-Hausdorff relation, the unitary transformation (6) can be rewritten in the form

$$
\hat{U}=e^{-\nu} e^{-\tau \hat{a}^{\dagger} \hat{b}^{\dagger}} e^{-\nu\left(\hat{a}^{\dagger} \hat{a}+\hat{b}^{\dagger} \hat{b}\right)} e^{\tau^{*} \hat{a} \hat{b}}
$$

where $\nu=\ln \cosh |\xi|$ and $\tau=\frac{\xi}{\xi} \tanh |\xi|$, so that the joint output state $|\Psi\rangle$ of the two modes can be expressed in the double-coherentstate basis $|a\rangle_{a}|\beta\rangle_{b}$, namely

$$
\begin{aligned}
\langle a, \beta \mid \Psi\rangle & =\frac{1}{\sqrt{1+z^{2}}}(\langle a, \beta|\hat{U}| 0,0\rangle+z\langle a, \beta|\hat{U}| m, 0\rangle) \\
& =\frac{1}{\sqrt{1+z^{2}}}\left(\left(1-|\tau|^{2}\right)^{\frac{1}{2}}+z \frac{\left(1-|\tau|^{2}\right)^{\frac{m+1}{2}}}{\sqrt{m !}}\right)
\end{aligned}
$$




$$
\times e^{-\left(|a|^{2}+|\beta|^{2}\right) / 2-\tau \alpha^{*} \beta^{*}+\tau^{*} \alpha \beta} .
$$

From Equation (14), we can easily write the reduced output state $\hat{\rho}$ obtained by tracing $|\Psi\rangle$ over the idler mode and paying attention to the non-orthogonality of coherent states. Using again the notation $\bar{a}=\left(a_{1}, \ldots a_{n}\right)^{T}$, we get

$$
\operatorname{tr}\left(\hat{\rho}^{n}\right)=\frac{\left(1-|\tau|^{2}\right)^{n}}{\pi^{n}\left(1+z^{2}\right)^{n}} \prod_{j=1}^{n} \int d^{2} a_{j}\left|1+c a_{j}^{m}\right|^{2} e^{-\bar{a}^{\dagger} M \bar{a}}
$$

where the matrix $M$ is defined as in Equation (3) and

$$
c=\frac{z}{\sqrt{m !}}\left(1-|\tau|^{2}\right)^{m / 2} \text {. }
$$

In order to bring this back to a Gaussian integral, we use the 'sources' trick, $^{23}$ exploiting the identity $x^{m} e^{-x^{2}}=\left.\frac{\partial^{m}}{\partial \lambda^{m}} e^{-x^{2}+\lambda x}\right|_{\lambda=0}$. Then, Equation (15) becomes

$$
\begin{aligned}
\operatorname{tr}\left(\hat{\rho}^{n}\right)= & \frac{\left(1-|\tau|^{2}\right)^{n}}{\pi^{n}\left(1+z^{2}\right)^{n}} \prod_{\partial \lambda}(n) \prod_{j=1}^{n} \int d^{2} a_{j} \\
& \times\left.\exp \left(-\bar{a}^{\dagger} M \bar{a}+\bar{a}^{\dagger} \bar{\lambda}+\bar{\lambda}^{\dagger} \bar{a}\right)\right|_{\bar{\lambda}=\overline{0}}
\end{aligned}
$$

where $\prod_{\partial \lambda}(n) \equiv \prod_{j=1}^{n}\left|1+c \partial^{m} / \partial \lambda_{j}^{m}\right|^{2}$ is a differential operator in the variables $\bar{\lambda}=\left(\lambda_{1}, \ldots \lambda_{n}\right)^{T}$. Note here that $\lambda_{j}$ and $\lambda_{j}^{*}$ are treated as independent variables, instead of their real and imaginary parts. The derivatives with respect to all $\lambda^{\prime}$ s have been pushed in front of the integrals in Equation (17), so that we get a Gaussian integral that is immediately calculable, resulting in

$$
\operatorname{tr}\left(\hat{\rho}^{n}\right)=\left.\frac{\operatorname{tr}\left(\hat{\rho}_{0}^{n}\right)}{\left(1+z^{2}\right)^{n}} \prod_{\partial \lambda}(n) \exp \left(\bar{\lambda}^{\dagger} N \bar{\lambda}\right)\right|_{\bar{\lambda}=\overline{0}},
$$

where $\operatorname{tr}\left(\hat{\rho}_{0}^{n}\right)$ is given by Equation (4) and corresponds to a vacuum input state $(z=0)$. We have defined here the circulant matrix $N=\left(1-|\tau|^{2 n}\right)^{m} M^{-1}$, with

$$
M^{-1}=\frac{1}{1-|\tau|^{2 n}}\left(\begin{array}{cccc}
1 & |\tau|^{2} & \ldots & |\tau|^{2(n-1)} \\
|\tau|^{2(n-1)} & 1 & \ldots & |\tau|^{2(n-2)} \\
\vdots & \vdots & \ddots & \vdots \\
|\tau|^{2} & |\tau|^{4} & \ldots & 1
\end{array}\right)
$$

Equation (18) can finally be re-expressed in a form that is suitable to the replica method (see Materials and Methods), which yields a nice expression for the output entropy

$$
S(z)=\frac{1}{1+z^{2}} S_{0}+\frac{z^{2}}{1+z^{2}} S_{m}
$$

Intriguingly, the entropy of the amplified state is thus a convex combination of the extremal points $S_{0}$ and $S_{m}$ with the exact same weights as if we had lost coherence between the components $|0\rangle$ and $|m\rangle$ of the input superposition. This is schematically pictured in Figure 1. We have also numerically verified this behaviour, which, to our knowledge, has never been observed before. This is illustrated in Figure 2, where we show that the entropy is a monotonically increasing function of the superposition parameter $z$ for fixed values of the squeezing parameter $\xi$. It must be stressed that this analytical result is highly non-trivial as we do not expect similar expressions for

$$
S_{0}=\stackrel{\bullet}{S}(0) \quad \stackrel{S(z)}{ } \quad \stackrel{S}{S_{m}}=S(z \rightarrow \infty)
$$

Figure 1. Generated entropy as a linear function over a onedimensional convex polytope. The von Neumann entropy $S(z)$, function of the superposition parameter $z$, is pictured by a point belonging to a one-dimensional convex polytope. The two extremal points correspond to the entropies $S_{0}$ and $S_{m}$, obtained by amplifying states $|0\rangle$ and $|m\rangle$, respectively. the entropy generated by other superpositions, such as $|1\rangle+z|2\rangle$ or $|0\rangle+z|1\rangle+z^{\prime}|2\rangle$. Another striking case is the amplification of a coherent state: although $|a\rangle$ is an infinite superposition of Fock states, the resulting entropy is $S_{0}$, just as for the vacuum state $|0\rangle$.

\section{DISCUSSION}

We have demonstrated that the replica method, a tool borrowed from other areas of physics, provides a new angle of attack to access the quantum entropies generated by Gaussian bosonic transformations, especially in cases where the symplectic formalism is useless. As a matter of fact, it did require considerable effort simply to show that the vacuum state minimises the entropy produced by Gaussian bosonic channels, as recently proven in ref. 24. The difficulty behind this proof was precisely that no diagonal representation of the output state $\hat{\rho}$ is available when non-Gaussian input states are considered. A similar problem is also at the heart of other-yet unproven-Gaussian entropic conjectures for bosonic channels. For example, determining the capacity of a multiple-access or broadcast Gaussian bosonic channel is pending on being able to access entropies when nonGaussian mixed input states are considered, see, e.g., refs 25-27. The replica method holds the promise to unblock these situations as it provides a generic trick to overcome the difficulty: $\operatorname{tr}\left(\hat{\rho}^{n}\right)$ is expressed for $n$ replicas of state $\hat{\rho}$ by solving Gaussian integrals, without ever accessing the eigenvalues of $\hat{\rho}$.

We have illustrated this procedure for the amplification of a superposition state of the form $|0\rangle+z|m\rangle$, which allowed us to unveil a remarkably simple behaviour for the entropy of the amplified state. The replica method thus clearly extends the limits of exactly computable entropies in comparison with the usual methods. Yet, one may question whether this method is sufficiently robust to address more complicated situations, such as arbitrary input states or general Gaussian transformations (other than the Bogoliubov transformation considered here). Even if a closed expression for $\operatorname{tr}\left(\hat{\rho}^{n}\right)$ can be found, taking its derivative with respect to $n$ is a task that may become impractical. Although it is uncertain whether we can apply the replica method in such cases, we nevertheless believe that this method remains valuable if the goal is to find a bound on the entropy (instead of its exact expression). Take, for example, a general input state $|\psi\rangle=\sum_{k} c_{k}|k\rangle$ in Fock space. Our current calculations have shown that applying a Bogoliubov transformation yields an output state whose moments can be written as $\operatorname{tr}\left(\hat{\rho}^{n}\right)=\operatorname{tr}\left(\hat{\rho}_{0}^{n}\right) T_{\psi}(n)$, where $\hat{\rho}_{0}$ is the output state that results from a vacuum input

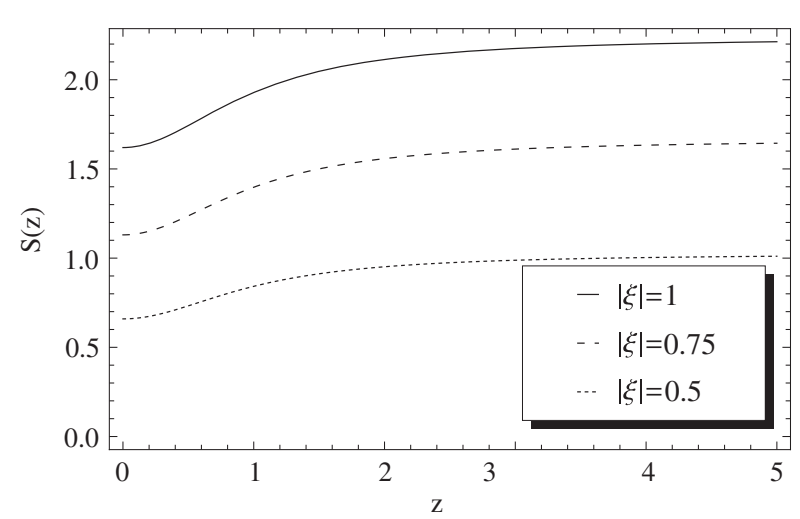

Figure 2. Amplification of a superposition of the vacuum and a single-photon state. Plot of the von Neumann entropy $S(z)$ as a function of the superposition parameter $z$ for $m=1$ and several values of the squeezing parameter $\xi$. As $S_{1}>S_{0}$, Equation (20) implies that the curve $S(z)$ always lies above $S_{0}$ for a given $\xi$. 
state and $T_{\psi}(n)$ is some analytical function of $n$, which encapsulates the actual input state $|\psi\rangle$. Similarly as in Equation (17), the function $T_{\psi}(n)$ can be written by applying some differential operator to the function $\prod_{j=1}^{n} f\left|\left(a_{j}\right)\right|^{2}$ with $f(a)=\sum_{k=0}^{\infty} c_{k} a^{k} / \sqrt{k !}$. The above expression for $\operatorname{tr}\left(\hat{\rho}^{n}\right)$, written in terms of $\operatorname{tr}\left(\hat{\rho}_{0}^{n}\right)$, is especially relevant in the context of Gaussian entropic conjectures. It can, for example, be linked to the property that the vacuum input minimises the output entropy of the Bogoliubov transformation, as shown in a forthcoming work.

Another possible extension of the present work would be to consider more general Gaussian transformations as well as mixed input states. A possibility is to use the Glauber-Sudarshan $P$ representation of the input and output mixed states (instead of their non-diagonal coherent-state basis decompositions), which may lead to a simpler way to express $\operatorname{tr}\left(\hat{\rho}^{n}\right)$. Our preliminary results indicate that the replica method is again promising here, for example, to compute (or lower bound) the output entropy of a beam splitter if the input state is mixed. This avenue will be further explored in a future work.

In conclusion, we anticipate that the replica method will become quite a valuable tool in order to reach a complete entropic characterisation of Gaussian bosonic transformations, or perhaps even solve pending conjectures on Gaussian bosonic channels. More generally, the present work underlines the usefulness of a tool that had been neglected so far in the fields of quantum optics and quantum information theory.

\section{MATERIALS AND METHODS}

Replica method

The replica method relies in general on the identity $\log Z=$ $\lim _{n \rightarrow 0}\left(Z^{n}-1\right) / n$. For our purposes, using $x \log x=\lim _{n \rightarrow 1}\left(x^{n}-x\right) /$ $(n-1)=\left.\frac{\partial}{\partial n}\left(x^{n}\right)\right|_{n=1}$, we may re-express the von Neumann entropy of a state $\hat{\rho}$ as

$$
S(\hat{\rho})=-\left.\frac{\partial \operatorname{tr}\left(\hat{\rho}^{n}\right)}{\partial n}\right|_{n=1}
$$

The trick is to find an analytical expression of $\operatorname{tr}\left(\hat{\rho}^{n}\right)$ as a function of the number of replicas $n \in \mathbb{N}^{*}$ and to compute its derivative at $n=1$, avoiding the need to diagonalise $\hat{\rho}$. This method also makes apparent the connection between the von Neumann entropy and other widely used measures of disorder, such as Tsallis and Rényi entropies. It has been used with great success in the context of spin glasses and quantum field theory, ${ }^{19,20,28-33}$ being justified based on the analyticity of $\operatorname{tr}\left(\hat{\rho}^{n}\right)$ in a neighbourhood of $n=1.29,30$ In the Supplementary Information, we discuss its validity using Hausdorff's moment problem and provide some easy but instructive examples from classical probability theory.

Applying the replica method to Equation (18)

The differential operator $\prod_{\partial \lambda}(n)$ appearing in Equation (18) can be expanded as

$$
\prod_{\partial \lambda}(n)=\sum_{k=0}^{n} c^{2 k} \prod_{2 k}(n)
$$

where each $\prod_{2 k}(n)$ contains $\left(\begin{array}{l}n \\ k\end{array}\right)^{2}$ terms that return a non-zero result when acting on $\exp \left(\bar{\lambda}^{\dagger} N \bar{\lambda}\right)$ and taking the value at $\bar{\lambda}=\overline{0}$, see Supplementary Information for details. The term with $k=0$ in Equation (22) is simply $\prod_{0}(n)=1$, so that taking $z=0$ trivially results into $\operatorname{tr}\left(\hat{\rho}^{n}\right)=\operatorname{tr}\left(\hat{\rho}_{0}^{n}\right)$, corresponding to the amplification of a vacuum state. The term with $k=n$ gives, when acting on the exponential of Equation (18),

$$
\left.\prod_{2 n}(n) \exp \left(\bar{\lambda}^{\dagger} N \bar{\lambda}\right)\right|_{\bar{\lambda}=\overline{0}}=m ! n \frac{1-|\tau|^{2 n}}{|\tau|^{2 n}} \mathrm{Li}_{-n}^{(m)}\left(|\tau|^{2 n}\right)
$$

where $\mathrm{Li}_{-n}^{(m)}\left(|\tau|^{2 n}\right)$ is defined in Equation (10). Thus, we recognise that this term is connected to the case of an input Fock state $|m\rangle$, something that can also be seen by taking the limit $z \rightarrow \infty$ in Equation (18). If we put all pieces together, we may re-express Equation (18) as

$$
\begin{aligned}
\operatorname{tr}\left(\hat{\rho}^{n}\right)= & \frac{\operatorname{tr} \hat{\rho}_{0}^{n}}{\left(1+z^{2}\right)^{n}}\left\{\left[1+z^{2}\left(\frac{1-|\tau|^{2}}{1-|\tau|^{2 n}}\right)^{m}\right]^{n}-z^{2 n}\left(\frac{1-|\tau|^{2}}{1-|\tau|^{2 n}}\right)^{m n}\right. \\
& \left.+F^{(m)}(n)+z^{2 n} \frac{\operatorname{tr} \hat{\rho}_{m}^{n}}{\operatorname{tr} \hat{\rho}_{0}^{n}}\right\}
\end{aligned}
$$

where $\hat{\rho}_{m}$ is the reduced output state resulting from the amplification of $|m\rangle$, and $F^{(m)}(n)$ is defined in the Supplementary Information. Now, applying the replica method (Equation (21)) to Equation (24), we get

$$
S(z)=\frac{1}{1+z^{2}} S_{0}+\frac{z^{2}}{1+z^{2}} S_{m}-\left.\frac{\partial}{\partial n} F^{(m)}(n)\right|_{n=1} .
$$

Finally, we prove in the Supplementary Information that the last term of the right-hand side of Equation (25) vanishes, so that it simplifies into Equation (20).

\section{ACKNOWLEDGEMENTS}

We thank R. García-Patrón, J. Schäfer and O. Oreshkov for useful discussions. This work was supported by the FRS-FNRS under Project No. T.0199.13 and by the Belgian Federal IAP programme under Project No. P7/35. CNG acknowledges financial support from Wallonia-Brussels International via the excellence grants programme.

\section{COMPETING INTERESTS}

The authors declare no conflict of interest.

\section{REFERENCES}

1. Leonhardt, U. Essential quantum optics: from quantum measurements to black holes (Cambridge Univ. Press, 2010).

2. Bogoliubov, N. N. A new method in the theory of superconductivity I. J. Exp. Theor. Phys. 34, 58-65 (1958)

3. Loudon, R. Non-classical effects in the statistical properties of light. Rep. Prog. Phys. 43, 913-949 (1980).

4. Loudon, R. \& Knight, P. L. Squeezed light. J. Mod. Opt. 34, 709-759 (1987).

5. Slusher, R. E., Hollberg, L. W., Yurke, B., Mertz, J. C. \& Valley, J. F. Observation of squeezed states generated by four-wave mixing in an optical cavity. Phys. Rev. Lett. 55, 2409-2412 (1985).

6. Wu, L. A., Kimble, H. J., Hall, J. L. \& Wu, H. Generation of squeezed states by parametric down conversion. Phys. Rev. Lett. 57, 2520-2523 (1986).

7. Fulling, S. A. Nonuniqueness of canonical field quantization in riemannian space-time. Phys. Rev. D 7, 2850-2862 (1973).

8. Davies, P. C. W. Scalar production in Schwarzschild and Rindler metrics. J. Phys. A 8, 609-616 (1975)

9. Unruh, W. G. Notes on black-hole evaporation. Phys. Rev. D 14, 870-892 (1976).

10. Hawking, S. Black hole explosions? Nature 248, 30-31 (1974).

11. Hawking, S. Particle creation by black holes. Comm. Math. Phys. 43, 199-220 (1975).

12. Bradler, K. \& Adami, C. Black holes as bosonic Gaussian channels. Phys. Rev. D 92 025030 (2015).

13. Weedbrook, C. et al. Gaussian quantum information. Rev. Mod. Phys. 84, 621-669 (2012)

14. Wehrl, A. General properties of entropy. Rev. Mod. Phys. 50, 221-260 (1978).

15. Caves, C. M. Quantum limits on noise in linear amplifiers. Phys. Rev. D 26, 1817-1839 (1982).

16. Holevo, A. S. \& Werner, R. F. Evaluating capacities of bosonic Gaussian channels. Phys. Rev. A 63, 032312 (2001)

17. Giovannetti, V., Guha, S., Lloyd, S., Maccone, L. \& Shapiro, J. H. Minimum output entropy of bosonic channels: a conjecture. Phys. Rev. A 70, 032315 (2004).

18. Garcia-Patron, R., Navarrete-Benlloch, C., Lloyd, S., Shapiro, J. H. \& Cerf., N. J. Majorization theory approach to the Gaussian channel minimum entropy conjecture. Phys. Rev. Lett. 108, 110505 (2012)

19. Mézard, M., Parisi, G. \& Virasoro., M. A. Spin glass theory and beyond (World Scientific, 1987).

20. Callan, C. \& Wilczek, F. On geometric entropy. Phys. Lett. B 333, 55-61 (1994).

21. Navarrete-Benlloch, C., Garcia-Patron, R., Shapiro, J. H. \& Cerf, N. J. Enhancing quantum entanglement by photon addition and subtraction. Phys. Rev. A $\mathbf{8 6}$ 012328 (2012) 
22. Olver F. W. J., Lozier D. W., Boisvert R. F. \& Clark C. W. (eds.) NIST Handbook of Mathematical Functions (Cambridge Univ. Press, National Institute of Standards and Technology, 2010).

23. Zeidler, E. Quantum Field Theory II Quantum Electrodynamics (Springer, 2009).

24. Giovannetti, V., Garcia-Patron, R., Cerf, N. J. \& Holevo, A. S. Ultimate classical communication rates of quantum optical channels. Nat. Photon. 8, 796-800 (2014).

25. Guha., S. Multiple-User Quantum Information Theory for Optical Communication Channels (PhD thesis. Massachusetts Institute of Technology, USA, 2008).

26. Yen, B. J. \& Shapiro, J. H. Multiple-access bosonic communications. Phys. Rev. A 72, 062312 (2005).

27. Guha, S., Shapiro, J. H. \& Erkmen, B. I. Classical capacity of bosonic broadcast communication and a minimum output entropy conjecture. Phys. Rev. A 76, 032303 (2007).

28. Holzhey, C., Larsen, F. \& Wilczek, F. Geometric and renormalized entropy in conformal field theory. Nucl. Phys. B 424, 443-467 (1994).

29. Callabrese, P. \& Cardy, J. Entanglement entropy and quantum field theory. J. Stat. Mech. 0406, 06002 (2004)
30. Callabrese, P. \& Cardy, J. Evolution of entanglement entropy in one-dimensional systems. J. Stat. Mech. 0504, 04010 (2005)

31. Ryu, S. \& Takayanagi, T. Aspects of holographic entanglement entropy. JHEP 0608, 045 (2006)

32. Berger, M. S. \& Buniy, R. V. Entanglement entropy and spatial geometry. JHEP 0807, 095 (2008).

33. Gagatsos, C. N., Karanikas, A. I. \& Kordas, G. Mutual information and Bose-Einstein condensation. Open Syst. Inf. Dyn. 20, 1350008 (2013).

cc (i) This work is licensed under a Creative Commons Attribution 4.0 International License. The images or other third party material in this article are included in the article's Creative Commons license, unless indicated otherwise in the credit line; if the material is not included under the Creative Commons license, users will need to obtain permission from the license holder to reproduce the material. To view a copy of this license, visit http://creativecommons.org/licenses/ by/4.0/

Supplementary Information accompanies the paper on the npj Quantum Information website (http://www.nature.com/npjqi) 